\title{
Holomorphic diffeomorphisms of complex semisimple Lie groups
}

\author{
Arpad Toth, Dror Varolin \\ Department of Mathematics, University of Michigan, East Hall, 525 East University Ave., \\ Ann Arbor, MI 48109-1109, USA
}

Oblatum 2-II-1999 \& 16-VI-1999 / Published online: 22 September 1999

\section{Introduction}

In this paper we study the group of holomorphic diffeomorphisms of a complex semisimple Lie group. These holomorphic diffeomorphism groups are infinite dimensional. To get additional information on these groups, we consider, for instance, the following basic problem: Given a complex semisimple Lie group $G$, a holomorphic vector field $X$ on $G$, and a compact subset $K \subset \subset G$, the flow of $X$, when restricted to $K$, is defined up to some nonzero time, and gives a biholomorphic map from $K$ onto its image. When can one approximate this map uniformly on $K$ by a global holomorphic diffeomorphism of $G$ ? One condition on a complex manifold which guarantees a positive solution of this problem, and which is possibly equivalent to it, is the so called density property, to be defined shortly. The main result of this paper is the following

Theorem. Every complex semisimple Lie group has the density property.

Let $M$ be a complex manifold and $\mathcal{X}_{\mathcal{O}}(M)$ the Lie algebra of holomorphic vector fields on $M$. Recall [V1] that a complex manifold is said to have the density property if the Lie subalgebra of $\mathcal{X}_{\mathcal{O}}(M)$ generated by its complete vector fields is a dense subalgebra. See Sect. 2 for the definition of completeness. Since $\mathcal{X}_{\mathcal{O}}(M)$ is extremely large when $M$ is Stein, the density property is particularly nontrivial in this case.

One of the two main tools underlying the proof of our theorem is the general notion of shears and overshears, introduced in [V3]. The condition of being a shear (again, see Sect. 2) is almost algebraic, and in fact is algebraic on Lie groups, facilitating the use of the second main tool, the representation theory of complex semisimple Lie groups. The latter is a complete and well known theory for which there are many references. We recommend especially $[\mathrm{FH}]$. We make use of a fair bit of the general theory. 
It is worth remarking that complex solvable Lie groups are biholomorphic to quotients of complex Euclidean space, and hence Stein solvable Lie groups, being biholomorphic to $\mathbb{C}^{n} \times\left(\mathbb{C}^{*}\right)^{k}$ have the density property if $n>1$ by one of the main results of [V1]. (It is not known if $\left(\mathbb{C}^{*}\right)^{k}$ has the density property, but it does have the similar volume density property; see [V1] for the definition.)

As already mentioned, a possibly equivalent definition (and definitely a consequence) of the density property is that the flow of any holomorphic vector field (even time dependent) on $M$ can be approximated by holomorphic diffeomorphisms locally uniformly on its domain of definition. This was used in [V2] to prove various results about (mostly Stein) manifolds with the density property. Since every complex semisimple Lie group is Stein, our main theorem and the results of [V2] imply several interesting corollaries, a few of which we state now. Let $G$ be a complex semisimple Lie group of complex dimension $n$.

Corollary 1. $G$ has an open subset which is biholomorphic to $\mathbb{C}^{n}$, i.e., a Fatou-Bieberbach domain of the first kind.

Corollary 2. $G$ is biholomorphic to one of its proper open subsets, i.e., it admits Fatou-Bieberbach domains of the second kind.

Corollary 3. Let $j: M \hookrightarrow G$ be a proper holomorphic embedding and let $E$ be a (possibly infinite) discrete subset of $G$. Then there exists another proper holomorphic embedding $j^{\prime}: M \hookrightarrow G$ such that $j^{\prime}(M) \supset E$.

In contrast, results of J. Winkelmann [W] allow us to conclude from Corollary 3 the following result.

Corollary 4. Let $M$ be a complex manifold such that there exists a proper holomorphic embedding $j: M \hookrightarrow G$. Then there exists another proper holomorphic embedding $j^{\prime}: M \hookrightarrow G$ such that for any $\varphi \in \operatorname{Diff}_{\mathcal{O}}(G)$, $\varphi \circ j(M) \neq j^{\prime}(M)$.

In Corollary 4 we denote the group of holomorphic diffeomorphisms of $G$ by $\operatorname{Diff}_{\mathcal{O}}(G)$ instead of the usual Aut $G$, since the latter has a standard meaning in the theory of Lie groups, and is liable to cause confusion. We even use the words "holomorphic diffeomorphisms" instead of "automorphisms" in an attempt to avoid possible confusion.

Corollary 4 is interesting because the density property guarantees that $\operatorname{Diff}_{\mathcal{O}}(G)$ is an extremely large group, and yet there still emerges a sort of holomorphic rigidity.

We wish to give a brief idea of how one proves these corollaries. More details may be found in [V2]. The main principle is that in the presence of the density property on a Stein manifold, one can construct holomorphic diffeomorphisms having any given jet at some point. Moreover, one can make these holomorphic diffeomorphisms arbitrarily close to the identity on a compact set whose holomorphic hull does not contain the source and target of the jet in question. With this, Corollary 1 follows from the existence of 
a holomorphic diffeomorphism with an attracting fixed point. Corollaries 2 and 3 are obtained by an inductive construction using the aforementioned jet theorem, which for the case of $\mathbb{C}^{n}$ has appeared in various papers (see the next paragraph for references.) To reiterate, Corollary 4 is a consequence of Corollary 3 and Winkelmann's results.

The first theorem about the density property is due to E. Andersén [A]. Answering a question of Rosay and Rudin [RR], he showed in this important paper that shears generate a dense subgroup of the group of holomorphic diffeomorphisms of $\mathbb{C}^{n}$ having Jacobian determinant 1. Although he was not explicit about it, the main idea was to prove that $\left(\mathbb{C}^{n}, d z_{1} \wedge \ldots . \wedge d z_{n}\right)$ has the volume density property. Shortly afterwards, Andersén and L. Lempert [AL] generalized the results of $[\mathrm{A}]$ to show that $\mathbb{C}^{n}$ has the density property. The results of Andersén and Lempert were used by F. Forstnerič and J.-P. Rosay [FR] to prove many results about approximation of biholomorphic mappings by holomorphic diffeomorphisms. The techniques have since had many interesting applications in the analytic geometry of $\mathbb{C}^{n}$. For surveys of some of these results, see $[\mathrm{F}]$ and $[\mathrm{R}]$.

The organization of the paper is as follows: In Sect. 2 we write down a few definitions and basic facts about some of the objects we will be making use of. This is also an opportunity to establish some notation. In Sect. 3 we study $\mathfrak{s l}(2, \mathbb{C})$-algebras, which are a key tool in our analysis. We establish an important result, which is responsible for "half" of the proof of our main theorem. In Sect. 4 we derive two consequences of the results of Sect. 3: that $\mathbb{P} S L(2, \mathbb{C})$ and the complex quadric $Q^{2}=\left\{x^{2}+y^{2}+z^{2}=1\right\} \subset \mathbb{C}^{3}$ have the density and volume density property. Some but not all of the most important ideas of our proof are contained here. The remainder of the paper is then devoted to proving one of four possible generalizations of the results of Sect. 4, namely our main result. The questions of the density property on homogeneous spaces and of the volume density property will be taken up in forthcoming papers. In Sect. 5 we state and prove a criterion which guarantees the density property. The proof relies heavily on the results of Sect. 3, and may be thought of as one possible completion of the story started there. In Sect. 6 we apply our criterion to prove the density property for all the adjoint groups. This is our way of easing the reader (and ourselves; our results were actually discovered in this order) into the fact that most of what is needed for applying the criterion to any complex semisimple Lie group is a "good" representation, which the adjoint representation happens to be. The (definition and) construction of a good representation of another sort is then the main part of Sect. 7, which also includes a (now straightforward) proof of the main theorem.

Let us end this introduction with several remarks. First of all, there is very little known about the nature of the density property. The results and methods of this paper suggest an interesting possibility: perhaps the density property is connected with two ideas; the existence of a few complete vector fields and (hopefully many) associated shears, and the representation theoretic properties of the Lie algebra of holomorphic vector fields relative 
to these shears. As will be seen below, shears can be thought of as highest weight vectors, and overshears "second highest"; this fact is a key point in the proof.

The other point worth making is the following: Possibly one of the main benefits of this paper is that it can be used to produce examples. More than just examples of the density property, one can construct examples of interesting dynamical systems on complex semisimple Lie groups. To the non-expert (e.g. us) of Lie theory the machinery used here might seem formidable. However, as is often the case with Lie groups, if one actually tries to work out an example, things become rather straightforward. Construction of shears and so on is easily made explicitly, so the expert in complex analysis or dynamics who wants to do such things will find that the paper may be deciphered rather easily. In fact, we were tempted to produce lists for such purposes, but decided against it for several reasons.

\section{Some background material}

\section{Holomorphic vector fields}

A holomorphic vector field $X$ on a complex manifold $M$ is a holomorphic section of $T^{1,0} M$, the holomorphic part of the complexified tangent bundle. we denote the set of holomorphic vector fields on $M$ by $\chi_{\mathcal{O}}(M)$. Since $T^{1,0} M$ is naturally isomorphic to the (real) tangent bundle $T M$, we can identify $X$ with a real vector field (which we continue to denote by $X$ ). As such, there is a flow $\varphi_{X}$ associated to $X$, which is defined on an open subset $\mathcal{U}$ of $\mathbb{R} \times M$ containing $\{0\} \times M$ in the following way: For $(t, p) \in \mathcal{U}$, $\varphi_{X}^{t}(p)=c(t)$, where $c:(-a, b) \rightarrow M$ is the unique maximal solution of the initial value problem

$$
\frac{d c}{d t}=X \circ c, \quad c(0)=p .
$$

It follows from general ODE theory that the map $p \rightarrow \varphi_{X}^{t}(p)$ is holomorphic.

We say that $X$ is complete if $U=\mathbb{R} \times M$, i.e., if for each $p \in M$ one can solve (*) for all $t \in \mathbb{R}$. In this case $\left\{\varphi_{X}^{t} \mid t \in \mathbb{R}\right\}$ is a one parameter group of holomorphic diffeomorphisms of $M$. We say that $X$ is $\mathbb{C}$-complete if both $X$ and $i X$ are complete. Define the $\mathbb{C}$-flow of $X$ to be

$$
g_{X}^{s+i t}(p):=\varphi_{X}^{s} \circ \varphi_{i X}^{t} .
$$

If $X$ is $\mathbb{C}$-complete, then $\left\{g_{X}^{\zeta} \mid \zeta \in \mathbb{C}\right\}$ defines a holomorphic $\mathbb{C}$-action. (To see this, use the fact that for holomorphic vector fields $X,[X, i X]=0$.) In this paper, we shall not consider vector fields which are complete but not $\mathbb{C}$-complete. Hence we shall sometimes drop the prefix $\mathbb{C}$-, and still refer to $g_{X}$ as the flow of $X$, even though it is defined for "complex time". 
With the operation $[X, Y]=X Y-Y X, \mathcal{X}_{\mathcal{O}}(M)$ forms a Lie algebra. We can generate a Lie subalgebra of $\mathcal{X}_{\mathcal{O}}(M)$ using complete vector fields on $M$. We shall call any vector field in the closure of this subalgebra completely generated. In general, this subalgebra will not consist of complete vector fields. However, completely generated vector fields have the extraordinary property that their flows can be approximated (in the locally uniform, and hence $\mathcal{C}^{k}$ topology) by holomorphic diffeomorphisms of $M$ [V1].

Suppose now that $M$ admits a nowhere vanishing holomorphic $n$-form $\omega$, where $n=\operatorname{dim}_{\mathbb{C}} M$. We call such a form a holomorphic volume element. We can use $\omega$ to define a map $\operatorname{div}_{\omega}: \mathcal{X}_{\mathcal{O}}(M) \rightarrow \mathcal{O}(M)$ called divergence (or $\omega$-divergence) by the relation

$$
\operatorname{div}_{\omega}(X) \omega=L_{X} \omega
$$

where $L_{X}$ is the Lie derivative along $X$ : if $\alpha$ is any tensor, then

$$
L_{X} \alpha:=\left.\frac{d}{d t}\right|_{t=0}\left(\varphi_{X}^{t}\right)^{*} \alpha .
$$

Since $L_{[X, Y]}=\left[L_{X}, L_{Y}\right]$, one can easily deduce that

$$
\operatorname{div}_{\omega}[X, Y]=X \operatorname{div}_{\omega} Y-Y \operatorname{div}_{\omega} X .
$$

If $\alpha$ is a differential form, then H. Cartan's formula $L_{X} \alpha=d\left(i_{X} \alpha\right)+i_{X} d \alpha$ (where $i_{X}$ is the contraction with $X$ ) shows that

$$
\operatorname{div}_{\omega}(X) \omega=d\left(i_{X} \omega\right)
$$

which makes divergence easy to compute. It is also easy to show that if $X \in \mathcal{X}_{\mathcal{O}}(M)$ and $f \in \mathcal{O}(M)$, then

$$
\operatorname{div}(f \cdot X)=X f+f \operatorname{div} X .
$$

We denote the kernel of $\operatorname{div}_{\omega}$ by $\mathcal{X}_{\mathcal{O}}(M, \omega)$. If $X \in \mathcal{X}_{\mathcal{O}}(M, \omega)$ we also call $X$ a divergence zero vector field. Finally, if $X$ belongs to the Lie subalgebra of $\mathcal{X}_{\mathcal{O}}(M, \omega)$ generated by complete vector fields in $\mathcal{X}_{\mathcal{O}}(M, \omega)$, we shall say that $X$ is divergence zero completely generated.

Example. Let $G$ be a complex Lie group, and let $V_{1}, \ldots V_{n}$ be any basis of left invariant vector fields. Let $\alpha_{1}, \ldots, \alpha_{n}$ be their dual left invariant one forms:

$$
\alpha_{j}\left(V_{k}\right)=\delta_{j k} .
$$

Set $\omega:=\alpha_{1} \wedge \ldots \wedge \alpha_{n}$. If $X=\sum f_{j} V_{j}$ is any holomorphic vector field on $G$, then

$$
\operatorname{div}_{\omega} X=\sum V_{j}\left(f_{j}\right)
$$

Note that $\mathcal{X}_{\mathcal{O}}(G, \omega)$ does not depend on the choice of $\omega$, (i.e., the choice of basis of left invariant vector fields) since any two such $\omega$ 's differ by a multiplicative constant.

\section{General shears}

In [V3] the following fundamental proposition was proved. 
Proposition 2.1. Let $X \in \mathcal{X}_{\mathcal{O}}(M)$ be $\mathbb{C}$-complete, and let $f \in \mathcal{O}(M)$. Then $f \cdot X$ is $\mathbb{C}$-complete if and only if $X^{2} f=0$.

One is thus naturally lead to study the function spaces

$$
\begin{aligned}
& I^{1}(X)=\{f \in \mathcal{O}(M) \mid X f=0\} \quad \text { and } \\
& I^{2}(X)=\left\{f \in \mathcal{O}(M) \mid X^{2} f=0\right\}
\end{aligned}
$$

consisting of (holomorphic) first and second integrals respectively.

Definition 2.2. Let $X \in \mathcal{X}_{\mathcal{O}}(M)$ be $\mathbb{C}$-complete. We call $f \cdot X$ an $X$-shear (resp. $X$-overshear) if $f \in I^{1}(X)$ (resp. $f \in I^{2}(X)$ ).

We will often refer to $X$-shears simply as shears, and similarly with overshears.

For a given complete vector field, the existence of first integrals is a classical problem and a highly nontrivial matter. The orbits of the vector field must sit fairly nicely together, generally speaking. For the existence of second integrals which are not first integrals, almost all the orbits must be biholomorphic to $\mathbb{C}$, and the orbit space must have extreme regularity [V3]. Nevertheless, in the case of complex (semisimple) Lie groups, many left invariant vector fields have a lot of first and second integrals, a fact we shall exploit thoroughly in this paper.

Finally, let us note that if $X$ has zero divergence, then $f \cdot X$ has divergence $X f$. Hence $X$-shears have zero divergence, and overshears may not.

\section{Finite dimensional representations of $\mathfrak{s l}(2, \mathbb{C})$}

There are many good references for this subject, for example $[\mathrm{FH}]$.

$\mathfrak{s l}(2, \mathbb{C})$ is the three dimensional complex Lie algebra with basis $\{E, F, H\}$ satisfying the commutation relations

$$
[H, E]=2 E \quad[H, F]=-2 F \quad[E, F]=H .
$$

It is an easy exercise to show that $\mathfrak{s l}(2, \mathbb{C})$ is simple, i.e., it is a non-Abelian Lie algebra with no proper ideals. One then needs relatively little effort to prove the following basic theorem.

Theorem 2.3. For every nonnegative integer $n$, there exists a unique irreducible representation $V^{(n)}$ of dimension $n+1$. This representation is of the form

$$
V^{(n)}=\bigoplus_{j=0}^{n} V_{n-2 j},
$$

where $V_{\alpha}$ is the one dimensional subspace of $V^{(n)}$ consisting of eigenvectors of $H$ with eigenvalue $\alpha$. 
It is a theorem of $\mathrm{H}$. Weyl that every representation of a simple Lie algebra (in fact, even semisimple, i.e., direct sum of simple Lie algebras) is completely reducible, i.e., a direct sum of irreducible representations. However, even though Weyl's theorem tells us that every finite dimensional representation of $\mathfrak{s l}(2, \mathbb{C})$ is completely reducible, there is still a natural question to be answered: given a representation of $\mathfrak{s t}(2, \mathbb{C})$, how do we write it as a sum of irreducibles? This is called the Clebsch-Gordan problem, the solution of which we will need only in the following very simple case.

\section{Theorem 2.4.}

1. $V^{(n)} \otimes V^{(2)}=V^{(n+2)} \oplus V^{(n)} \oplus V^{(n-2)} \quad(n \geq 2)$

2. $V^{(1)} \otimes V^{(2)}=V^{(3)} \oplus V^{(1)}$

3. $V^{(0)} \otimes V^{(2)}=V^{(2)} \quad$ (This is the trivial case.)

\section{3. $\mathfrak{s l}(2, \mathbb{C})$ algebras}

An $\mathfrak{s l}(2, \mathbb{C})$-algebra is an algebra which is also an $\mathfrak{s l}(2, \mathbb{C})$-representation, such that the $\mathfrak{s l}(2, \mathbb{C})$-action is Leibniz with respect to the algebra multiplication. In this paper we shall consider only locally finite dimensional $\mathfrak{s l}(2, \mathbb{C})$ algebras, i.e, finitely generated $\mathfrak{s l}(2, \mathbb{C})$-algebras which are direct sums of finite dimensional (irreducible) representations.

Every locally finite dimensional $\mathfrak{s l}(2, \mathbb{C})$-algebra $A$ admits a natural $\mathbb{Z}_{2^{-}}$ grading $A=A_{0} \oplus A_{1}$ (this means $A_{i} \cdot A_{j} \subset A_{i+j}$ for $i, j \in \mathbb{Z}_{2}$ ) where $A_{0}$ (resp. $A_{1}$ ) is generated (as a vector space) by the elements of even (resp. odd) weight in $A$. Note that $A_{0}$ is a subalgebra.

Important example If $M$ is a $k$-dimensional representation of $\mathfrak{s t}(2, \mathbb{C})$, let $A=\mathbb{C}[M]$ be the algebra of polynomials on $M . A$ admits a natural $\mathfrak{s l}(2, \mathbb{C})$ action coming from the one on $M$, which may be described as follows: If $L \in \mathfrak{s l}(2, \mathbb{C})$ and $a^{n}:=a_{1}^{n_{1}} \cdot \ldots \cdot a_{k}^{n_{k}}$ is a monomial in $A$, then

$$
L\left(a^{n}\right)=\sum_{j=1}^{k} n_{j} a^{n-e_{j}} L\left(a_{j}\right) .
$$

Alternatively, we could describe the action analytically as follows:

$$
(L f)(a)=\left.\frac{d}{d t}\right|_{t=0} f(\exp (t L) \cdot a) .
$$

From now on, let $A$ be a commutative locally finite dimensional $\mathfrak{s l}(2, \mathbb{C})$ algebra with unit. We define the corresponding vector field algebra

$$
\mathcal{X}(A):=A \otimes \mathfrak{s l}(2, \mathbb{C}) .
$$


$\mathcal{X}(A)$ becomes a Lie algebra when endowed with the bilinear bracket operation defined on generators by

$[f \otimes X, g \otimes Y]:=f \cdot g \otimes[X, Y]+(f \cdot X g) \otimes Y-(g \cdot Y f) \otimes X$

$$
f, g \in A, \quad X, Y \in \mathfrak{s l}(2, \mathbb{C}) .
$$

$\mathcal{X}(A)$ is an $\mathfrak{s l}(2, \mathbb{C})$ module with the $\mathfrak{s l}(2, \mathbb{C})$-action coming from the adjoint action of the subalgebra $1 \otimes \mathfrak{s l}(2, \mathbb{C})$ :

$$
X(\xi)=[1 \otimes X, \xi] \quad X \in \mathfrak{s l}(2, \mathbb{C}), \xi \in \mathcal{X}(A) .
$$

Actually, $\mathcal{X}(A)$ is also locally finite dimensional, and hence admits its own $\mathbb{Z}_{2}$-grading

$$
\mathcal{X}(A)=\mathcal{X}_{0}(A) \oplus \mathcal{X}_{1}(A) .
$$

Moreover, since $\mathfrak{s l}(2, \mathbb{C})$ has only even weights, we have

$$
\mathcal{X}_{0}(A)=A_{0} \otimes \mathfrak{s l}(2, \mathbb{C}) \quad \text { and } \quad X_{1}(A)=A_{1} \otimes \mathfrak{s l}(2, \mathbb{C}) .
$$

Definition 3.1. Let div $: \mathcal{X}(A) \rightarrow A$ be defined by

$$
\operatorname{div}(e \otimes E+h \otimes H+f \otimes F):=E e+H h+F f .
$$

Let $\mathcal{X}^{*}(A)$ denote the kernel of div.

It is easy to show that

\section{Lemma 3.2.}

$$
\operatorname{div}\left[\xi_{1}, \xi_{2}\right]=\xi_{1} \operatorname{div} \xi_{2}-\xi_{2} \operatorname{div} \xi_{1}
$$

It follows from this lemma that div is a homomorphism of $\mathfrak{s l}(2, \mathbb{C})$-modules, and hence $X^{*}(A)$ is itself an $\mathfrak{s l}(2, \mathbb{C})$-module. The lemma also implies that $X^{*}(A)$ is a subalgebra of $\mathcal{X}(A)$. Hence it has the natural $\mathbb{Z}_{2}$-grading

$$
\chi^{*}(A)=X_{0}^{*}(A) \oplus \mathcal{X}_{1}^{*}(A),
$$

which further satisfies

$$
\mathcal{X}_{0}^{*}(A)=\mathcal{X}^{*}(A) \cap \mathcal{X}_{0}(A) \quad \text { and } \quad \mathcal{X}_{1}^{*}(A)=\mathcal{X}^{*}(A) \cap \mathcal{X}_{1}(A) .
$$

Let $A^{(n)}$ be an irreducible submodule of $A$ of highest weight $n$, and let $d i v_{n}$ be the restriction of $\operatorname{div}$ to $A^{(n)} \otimes \mathfrak{s l}(2, \mathbb{C})$. By Theorem 2.4,

$$
A^{(n)} \otimes \mathfrak{s l}(2, \mathbb{C}) \cong V^{(n+2)} \oplus V^{(n)} \oplus V^{(n-2)} .
$$

Lemma 3.3. With the notation above,

(i) div $v_{n}$ is surjective for $n>0$, and di $v_{0} \equiv 0$.

(ii) $X^{*}(A) \cap\left(A^{(n)} \otimes \mathfrak{s l}(2, \mathbb{C})\right)= \begin{cases}V^{(n+2)} \oplus V^{(n-2)} & \text { for } n \geq 2 \\ V^{(3)} & \text { for } n=1 \\ 1 \otimes \mathfrak{s l}(2, \mathbb{C}) & \text { for } n=0\end{cases}$ 
Proof. Let $v \in A^{(n)}$ be a highest weight vector, and let $\xi:=2 F v \otimes E+$ $n v \otimes H$. It is easy to verify that $E \cdot \xi(=[1 \otimes E, \xi])=0$ and that $\operatorname{div} \xi=$ $n(n+2) v$. Hence the highest weight vector $\xi$ of $V^{(n)}$ in $A^{(n)} \otimes \mathfrak{s l}(2, \mathbb{C})$ is mapped by div onto the highest weight vector $n(n+2) v$. The lemma now follows from the fact that div is a mapping of $\mathfrak{s t}(2, \mathbb{C})$-modules.

Definition 3.4. If $f \in A$ and $X \in \mathfrak{s l}(2, \mathbb{C})$, then $f \otimes X$ is called a shear (resp. overshear) if $X f=0$ (resp. $\left.X^{2} f=0\right)$.

We can now state the main result of this section.

Theorem 3.5. $\mathcal{X}_{0}(A)$ (resp. $\left.\mathcal{X}_{0}^{*}(A)\right)$ is generated ${ }^{1}$ by overshears (resp. shears).

Proof. It suffices to consider the case when $A=A^{(2 k)}$. Let $v \in A^{(2 k)}$ be a highest weight vector. Then

$$
v \otimes E, F v \otimes E \text { and } F^{k} v \otimes H
$$

are overshears, the first and third being shears. Since $A^{(2 k)} \otimes \mathfrak{s l}(2, \mathbb{C})$ and its div zero subalgebra are generated by their weight zero subspaces, the lemma is proved if we can show that

$$
F^{k+1}(v \otimes E), F^{k}(F v \otimes E) \text { and } F^{k} v \otimes H
$$

are linearly independent. However, an easy induction argument shows that

$$
F^{k+1}(v \otimes E)=F^{k+1} v \otimes E-(k+1) F^{k} v \otimes H+k(k+1) F^{k-1} v \otimes F
$$

and

$$
F^{k}(F v \otimes E)=F^{k+1} v \otimes E-k F^{k} v \otimes H+k(k-1) F^{k-1} v \otimes F,
$$

from which the independence of these three vectors is easily verified.

\section{Two preliminary examples}

We turn now to two applications of Theorem 3.5. These are based on the fact that, in some special situations, there are locally finite dimensional $\mathfrak{s l}(2, \mathbb{C})$-algebras $A$ of interest whose odd part is trivial: $A=A_{0}$. While this situation is rare as far as our needs go, it does happen in a few cases. We discuss two of these now.

\section{The complex Lie group $\mathbb{P} S L(2, \mathbb{C})$}

Let us begin with $S L(2, \mathbb{C})$, which we can think of as the set of $2 \times 2$ matrices of determinant 1 :

$$
S L(2, \mathbb{C})=\left\{\left(\begin{array}{ll}
a & b \\
c & d
\end{array}\right) ; a d-b c=1\right\} .
$$

\footnotetext{
1 as an $\mathfrak{s t}(2, \mathbb{C})$-module, and hence as a Lie algebra
} 
The group $\mathbb{P} S L(2, \mathbb{C})$ is just the quotient of $S L(2, \mathbb{C})$ by its center $\{ \pm I d\}$. The space $T_{I d}$ tangent to $S L(2, \mathbb{C})$ (hence $\mathbb{P} S L(2, \mathbb{C})$ ) at the identity will be identified with the set of traceless matrices:

$$
T_{I d}=\mathfrak{s l}(2, \mathbb{C})=\left\{\left(\begin{array}{ll}
a & b \\
c & d
\end{array}\right) ; a+d=0\right\} .
$$

It has the structure of a Lie algebra, where the Lie bracket is the commutator of two matrices: $\left[A, A^{\prime}\right]=A A^{\prime}-A^{\prime} A$. The standard basis of $\mathfrak{s l}(2, \mathbb{C})$ is $\{e, f, h\}$, where

$$
e=\left(\begin{array}{ll}
0 & 1 \\
0 & 0
\end{array}\right) \quad h=\left(\begin{array}{rr}
1 & 0 \\
0 & -1
\end{array}\right) \quad f=\left(\begin{array}{ll}
0 & 0 \\
1 & 0
\end{array}\right) .
$$

We define an action $S L(2, \mathbb{C}) \times \mathfrak{s l}(2, \mathbb{C}) \rightarrow \mathfrak{s l}(2, \mathbb{C})$ of $S L(2, \mathbb{C})$ on its Lie algebra as follows:

$$
(g, X) \mapsto A d_{g}(X):=g X g^{-1} .
$$

Then $A d: S L(2, \mathbb{C}) \ni g \mapsto A d_{g} \in \operatorname{End} \mathfrak{s l}(2, \mathbb{C})$ is a representation of $S L(2, \mathbb{C})$ on $\mathfrak{s l}(2, \mathbb{C})$, called the adjoint representation. It is not hard to prove that the kernel of $A d$ is precisely $\{ \pm I d\}$. Thus $A d$ factors through a faithful representation of $\mathbb{P} S L(2, \mathbb{C})$, which is also denoted $A d$. The differential of $A d$ at $I d$ is denoted $a d: \mathfrak{s l}(2, \mathbb{C}) \rightarrow$ End $\mathfrak{s l}(2, \mathbb{C})$. It is easy to show that $\operatorname{ad}(X)(Y)=[X, Y]$. In the $\{e, h, f\}$ basis, one has

$\operatorname{ad}(e)=\left(\begin{array}{rrr}0 & -2 & 0 \\ 0 & 0 & 1 \\ 0 & 0 & 0\end{array}\right) \quad a d(h)=\left(\begin{array}{rrr}2 & 0 & 0 \\ 0 & 0 & 0 \\ 0 & 0 & -2\end{array}\right) \operatorname{ad}(f)=\left(\begin{array}{rrr}0 & 0 & 0 \\ -1 & 0 & 0 \\ 0 & 2 & 0\end{array}\right)$.

It follows that $\operatorname{Ad}(\mathbb{P} S L(2, \mathbb{C}))$ is a subgroup of $S L(\mathfrak{s l}(2, \mathbb{C}))$, which can further be shown to be closed. Thus, via $A d, \mathbb{P} S L(2, \mathbb{C})$ is a properly embedded submanifold of End $\mathfrak{s l}(2, \mathbb{C})\left(\cong \mathbb{C}^{9}\right)$. We denote by $\mathcal{O}_{A f f}(\mathbb{P} S L(2, \mathbb{C}))$ the set of restrictions of polynomials on $\operatorname{End} \mathfrak{s l}(2, \mathbb{C})$ to $\mathbb{P} S L(2, \mathbb{C})$. By Cartan's Theorem A, $\mathcal{O}_{A f f}(\mathbb{P} S L(2, \mathbb{C}))$ is a dense subset of $\mathcal{O}(\mathbb{P} S L(2, \mathbb{C}))$.

We can construct vector fields on End $\mathfrak{s l}(2, \mathbb{C})$ from elements of $\mathfrak{s l}(2, \mathbb{C})$ as follows (see also the example in Sect. 3): If $X \in \mathfrak{s l}(2, \mathbb{C})$, then define $\vec{X} \in \mathcal{X}_{A f f}(\operatorname{End} \mathfrak{s l}(2, \mathbb{C}))$ by

$$
\vec{X} f(m):=\left.\frac{d}{d t}\right|_{t=0} f(\operatorname{Ad}(\exp (t X)) \cdot m), \quad f \in \mathcal{O}(\operatorname{End} \mathfrak{s l}(2, \mathbb{C})),
$$

where $\cdot$ is composition of linear operators. Of course, $\vec{X}$ is a complete vector field on End $\mathfrak{s l}(2, \mathbb{C})$ which is tangent to $\operatorname{Ad}(\mathbb{P S L}(2, \mathbb{C}))$.

Next, let $x_{1}, x_{2}, x_{3}$ be any basis of $\mathfrak{s l}(2, \mathbb{C})$, and $\xi_{1}, \xi_{2}$ and $\xi_{3}$ its dual basis. In terms of this basis, we write $A d(X) x_{j}=\sum X_{j k} x_{k}$. Identifying End $\mathfrak{s l}(2, \mathbb{C})$ with its dual, we take as basis of linear functionals on End $\mathfrak{s l}(2, \mathbb{C}) \cong \mathfrak{s l}(2, \mathbb{C}) \otimes(\mathfrak{s l}(2, \mathbb{C}))^{*}$ the vectors $\left\{x_{i} \otimes \xi_{j} \mid 1 \leq i, j \leq 3\right\}$. Let $m_{i j}:=x_{i} \otimes \xi_{j}(m)$. 


\section{Proposition 4.1.}

$$
\vec{x}\left(x_{i} \otimes \xi_{j}\right)=\operatorname{ad}(x)\left(x_{i}\right) \otimes \xi_{j}=\left[x, x_{i}\right] \otimes \xi_{j} .
$$

Proof. Let $X^{t}:=\exp (t x)$. Then

$$
\begin{aligned}
x_{i} \otimes \xi_{j}\left(A d\left(X^{t}\right) m\right)=\sum_{k}\left(X^{t}\right)_{i k} m_{k j} & =\left(\sum_{k}\left(X^{t}\right)_{i k} x_{k}\right) \otimes \xi_{j}(m) \\
& =\left(A d\left(X^{t}\right) x_{i}\right) \otimes \xi_{j}(m) .
\end{aligned}
$$

Differentiating yields the result.

Now, $A=\mathcal{O}_{A f f}(\mathbb{P} S L(2, \mathbb{C}))$ is a locally finite dimensional $\mathfrak{s l}(2, \mathbb{C})$ algebra, which is a dense subalgebra of $\mathcal{O}(\mathbb{P S L}(2, \mathbb{C}))$. If we choose the basis $\{e, f, h\}$ for $\mathfrak{s l}(2, \mathbb{C})$, then $\vec{h}(e \otimes \xi)=2 e \otimes \xi, \vec{h}(f \otimes \xi)=-2 f \otimes \xi$, and $\vec{h}(h \otimes \xi)=0$. Hence $A=A_{0}$. Since $\mathcal{X}(A)=\mathcal{O}_{A f f} \otimes\{\vec{x} ; x \in$ $\mathfrak{s l}(2, \mathbb{C})\}\left(\right.$ resp. $\left.X^{*}(A)\right)$ is a dense Lie subalgebra of $\mathcal{X}_{\mathcal{O}}(\mathbb{P} S L(2, \mathbb{C}))$ (resp. $\mathcal{X}_{\mathcal{O}}(\mathbb{P} S L(2, \mathbb{C}), \omega)$ where $\omega$ is any right invariant volume element on $\mathbb{P} S L(2, \mathbb{C})$ ), we conclude that

Theorem 4.2. $\mathbb{P} S L(2, \mathbb{C})$ has the density and volume density property.

\section{The complexified sphere $Q^{2}$}

The quadric $Q^{2}$ is the closed 2 dimensional submanifold of $\mathbb{C}^{3}$ defined by

$$
Q^{2}:=\left\{x^{2}+y^{2}+z^{2}=1\right\} .
$$

By analogy with the real 2-sphere, one constructs three complete vector fields on $Q^{2}$, namely

$$
\begin{aligned}
& X(x, y, z)=z \partial_{y}-y \partial_{z}, \quad Y(x, y, z)=x \partial_{z}-z \partial_{x}, \text { and } \\
& Z(x, y, z)=y \partial_{x}-x \partial_{y} .
\end{aligned}
$$

The commutation relations

$$
[X, Y]=Z, \quad[Z, X]=Y, \quad \text { and } \quad[Y, Z]=X
$$

are easily verified, from which we get a subalgebra of $\chi_{\mathcal{O}}\left(Q^{2}\right)$ isomorphic to $\mathfrak{s o}(3, \mathbb{C})$. The basis $\{X, Y, Z\}$ of $\mathfrak{s o}(3, \mathbb{C})$ is particularly bad for us because the orbits of $X, Y$ and $Z$ are all $\mathbb{C}^{*}$ 's, and thus $X, Y$ and $Z$ have no nontrivial overshears (i.e., overshears which are not shears). Fortunately, $\mathfrak{s o}(3, \mathbb{C})$ and $\mathfrak{s l}(2, \mathbb{C})$ are isomorphic as Lie algebras, with the isomorphism given by

$$
E=X+i Y, \quad H=2 i Z, \quad F=-X+i Y .
$$

It can be checked directly that $E, H$ and $F$ are complete vector fields, but this simple change of basis suggests a change of coordinates in the ambient space $\mathbb{C}^{3}$. Set

$$
e=x+i y, \quad h=2 i z, \quad f=-x+i y .
$$


Then

$$
Q^{2}=\left\{4 e f+h^{2}=-4\right\} .
$$

With these changes of coordinates, it is easy to verify that

$$
E=h \partial_{f}-2 e \partial_{h}, \quad F=2 f \partial_{h}-h \partial_{e}, \quad \text { and } \quad H=2 e \partial_{e}-2 f \partial_{f} .
$$

We thus have a subalgebra of $\mathcal{X}_{\mathcal{O}}\left(Q^{2}\right)$ isomorphic to $\mathfrak{s l}(2, \mathbb{C})$. Moreover,

$$
H e=2 e, \quad H h=0 \quad \text { and } \quad H f=-2 f .
$$

As in the case of $\mathbb{P} S L(2, \mathbb{C})$, let $A:=\mathcal{O}_{A f f}\left(Q^{2}\right)$ be the set of restrictions of polynomials in $\mathbb{C}^{3}$ to $Q^{2}$. Then $A=A_{0}$, and since $\mathcal{O}_{A f f}\left(Q^{2}\right)$ is a dense subalgebra of $\mathcal{O}\left(Q^{2}\right)$, Theorem 3.5 implies that $\mathcal{X}(A)=A \otimes \operatorname{span}\{E, H, F\}$ is generated by overshears. Similarly, $\mathcal{X}^{*}(A)$ is generated by shears. Let

$$
\omega=x d y \wedge d z+y d z \wedge d x+z d x \wedge d y .
$$

Since clearly $\mathcal{X}(A)$ is a dense subset of $\mathcal{X}_{\mathcal{O}}\left(Q^{2}\right)$ and $\mathcal{X}^{*}(A)$ is a dense subalgebra of $\mathcal{X}_{\mathcal{O}}\left(Q^{2}, \omega\right)$, we obtain

Theorem 4.3. $Q^{2}$ has the density property, and $\left(Q^{2}, \omega\right)$ has the volume density property.

\section{A criterion for the density property}

At the level of $\mathfrak{s t}(2, \mathbb{C})$ algebras, one cannot conclude in general that overshears generate $\mathcal{X}(A)$ for any locally finite dimensional $\mathfrak{s l}(2, \mathbb{C})$-algebra $A$. However, with some additional information on $A$, one can push "complete generation" through. The needed information cannot be found at the algebraic level. (We shall elaborate momentarily.) We thus pass to a more concrete situation, which we now describe.

Let $M$ be a Stein manifold, which we think of as already embedded in some $\mathbb{C}^{n}$. Suppose that $\mathcal{X}_{\mathcal{O}}(M)$ contains a Lie subalgebra $\mathfrak{g}$ which is semisimple and consists of complete vector fields, and which further has the property that $\mathcal{O}(M) \cdot \mathfrak{g}=\mathcal{X}_{\mathcal{O}}(M)$. (Note that we don't ask for $M$ to be parallelized by $\mathfrak{g} ; \operatorname{dim} \mathfrak{g}$ may be greater than $\operatorname{dim}_{\mathbb{C}} M$.) Let $\mathcal{O}_{A f f}(M)$ denote the set of restrictions to $M$ of polynomials in $\mathbb{C}^{n}$, and write $\mathcal{X}_{A f f}(M):=\mathcal{O}_{A f f}(M) \cdot \mathfrak{g}$. Then $\mathcal{X}_{A f f}(M)$ is a dense subset of $\mathcal{X}_{\mathcal{O}}(M)$ in the locally uniform topology. We abuse notation and write $x_{i} \in \mathcal{O}_{A f f}(M)$ for the restriction of the coordinate function $x_{i} \in \mathcal{O}\left(\mathbb{C}^{n}\right)$. We need one last assumption, namely that there is a Cartan subalgebra $\mathfrak{h}$ of $\mathfrak{g}$ which preserves the space of linear functions, i.e., linear combinations of the $x_{i}$. The main result of this section is the following.

Theorem 5.1. Suppose that for each $X \in \mathfrak{g}$ and each $x_{i}$, the vector field $x_{i} X$ is completely generated. Then $\mathcal{X}_{A f f}(M)$ is generated by complete vector fields. Thus, in particular, $M$ has the density property. 
Proof. Clearly it suffices to check that for every monomial $f \in \mathcal{O}_{A f f}(M)$ and $X \in \mathfrak{g}$ a weight vector relative to some Cartan subalgebra $\mathfrak{h}, f X$ is completely generated. In this case, $X$ lies in some subalgebra of $\mathfrak{g}$ which is isomorphic to $\mathfrak{s l}(2, \mathbb{C})$. We fix a standard basis $\{E, H, F\}$ of this subalgebra, and choose coordinates in $\mathbb{C}^{n}$ so that each $x_{i}$ is a weight vector of $H$. We may further assume that $f$ is the restriction to $M$ of a monomial, hence itself a weight vector.

Note that if each $x_{i}$ has even weight, or if $f$ has even weight, then there is nothing to prove; the result is covered by Theorem 3.5. Hence we may assume without loss of generality that $x_{1}$ has odd weight $\lambda$ and that $f=x_{1} \cdot g$ where $g \in \mathcal{O}_{A f f}(M)$ has even weight $2 k$. Moreover, we note that it suffices to assume that $X=E, H$ or $F$.

Case 1: $(X=H)$ Then

$$
\left[g H, x_{1} H\right]=(\lambda-2 k) x_{1} g H=(\lambda-2 k) f H .
$$

Since $g H$ has even weight, it is generated by shears. The result follows from the fact that $\lambda-2 k$ is odd and hence not zero.

Case 2: $(X=E)$ Then

$$
\left[g H, x_{1} E\right]=g H\left(x_{1}\right) E+f[H, E]-x_{1} E(g) H=(\lambda+2) f E-x_{1} E(g) H .
$$

As in Case $1, g H$ is generated by shears. By Case $1, x_{1} E(g) H$ is completely generated. this case now follows from the fact that $\lambda+2$ is odd and hence not zero.

The case where $X=F$ is handled in a fashion similar to Case 2 .

To elaborate on the first paragraph of this section, whenever we show in this note that the hypotheses of Theorem 5.1 hold, we will have verified that $x_{i} X$ is generated by overshears. In this case, one can say (trivially) that $\mathcal{X}(A)$ is generated by shears. We cannot say, however, that in the most general situation it is always possible to verify these hypotheses. One may need to use complete vector fields which are not generated by shears associated to $\mathfrak{g}$.

\section{Adjoint groups}

In Sect. 4 we proved that $\mathbb{P} S L(2, \mathbb{C})$ has the density property. In this section we use Theorem 5.1 to extend this result to all the so-called adjoint groups; the (semi-simple) Lie groups with trivial center.

Let $G$ be a complex Lie group, and denote the operations of left and right multiplication with $g$ by $L_{g}$ and $R_{g}$ respectively. Let $\mathfrak{g}:=T_{e}(G)$, where $e \in G$ is the group identity. Define $A d: G \rightarrow$ End $\mathfrak{g}$ by

$$
g \mapsto A d_{g}:=d\left(L_{g} \circ R_{g^{-1}}\right)(e) .
$$


Clearly $A d$ is a representation of $G$ on $\mathfrak{g}$, and as is well known, $A d$ is faithful if and only if $G$ is a semi-simple Lie group which has trivial center. Moreover, in this case $\operatorname{Ad}(G)$ is a closed subgroup of $S L(\mathfrak{g})$, and hence $A d$ gives a proper holomorphic embedding of $G$ in End $\mathfrak{g} \cong \mathbb{C}^{n \times n}$, where $n=\operatorname{dim} \mathfrak{g}=\operatorname{dim}_{\mathbb{C}} G$. Finally, if we denote the derivative at $e$ of $A d: G \rightarrow$ End $\mathfrak{g}$ by ad $: \mathfrak{g} \rightarrow$ End $\mathfrak{g}$, then it is easy to show that $\operatorname{ad}(X)(Y)=[X, Y]$.

To every $X \in \mathfrak{g}$, we can associate a complete holomorphic vector field $\vec{X}$ on End $\mathfrak{g}$ defined by

$$
\vec{X} f(m)=\left.\frac{d}{d t}\right|_{t=0} f\left(A d_{\exp (t X)} \cdot m\right) \quad f \in \mathcal{O}(\text { End } \mathfrak{g}),
$$

where $\cdot$ is composition of linear operators. Evidently $\vec{X}$ is $\mathbb{C}$-complete.

Proposition 6.1. $\vec{X}$ is tangent to $\operatorname{Ad}(G)$.

Proof. Let $f$ be a (holomorphic) defining function for $A d(G)$ in a neighborhood $U$ of $A d_{g}$ in End $\mathfrak{g}$. Then

$$
\begin{aligned}
(d f \vec{X})_{A d_{g}}=\vec{X} f\left(A d_{g}\right) & =\left.\frac{d}{d t}\right|_{t=0} f\left(A d_{\exp (t X)} A d_{g}\right) \\
& =\left.\frac{d}{d t}\right|_{t=0} f\left(A d_{\exp (t X) g}\right)=0,
\end{aligned}
$$

since, for $t$ small enough, $A d_{\exp (t X) g} \in A d(G) \cap U$.

Let us now consider the algebra $\mathbb{C}[$ End $\mathfrak{g}]$ of polynomials on End $\mathfrak{g} \cong$ $\mathfrak{g} \otimes \mathfrak{g}^{*}$. This is the full symmetric algebra on $n^{2}$ elements. We use as a set of generators $\left\{x_{i} \otimes \xi_{j} \mid 1 \leq i, j \leq n\right\}$ where $\left\{x_{i} \mid 1 \leq i \leq n\right\}$ is a basis of $\mathfrak{g}$, and $\left\{\xi_{i} \mid 1 \leq i \leq n\right\}$ its dual basis. By identifying End $\mathfrak{g}$ with its dual, we think of the elements $x_{i} \otimes \xi_{j}$ as coordinate functions on End $\mathfrak{g}$. Then, using exactly the same ideas as in Proposition 4.1, one proves the following.

Proposition 6.2. Let $x, X \in \mathfrak{g}$ and $\xi \in \mathfrak{g}^{*}$. Then

$$
\vec{X}(x \otimes \xi)=[X, x] \otimes \xi .
$$

We can now state and prove our main result of this section.

Theorem 6.3. Every adjoint group has the density property.

The proof of this theorem is an immediate corollary of Theorem 5.1 and the following

Lemma 6.4. Let $G$ be an adjoint group with Lie algebra $\mathfrak{g}$. Then for each $x_{1}, x_{2} \in \mathfrak{g}$ and $\xi \in \mathfrak{g}^{*},\left(x_{1} \otimes \xi\right) \vec{x}_{2} \in X_{\mathcal{O}}($ End $\mathfrak{g})$ is generated by overshears. 
Proof. Since every adjoint group is a product of adjoint groups whose Lie algebras are simple, we may assume that $\mathfrak{g}$ is simple. Fix a Cartan subalgebra $\mathfrak{h}$ of $\mathfrak{g}$. We write

$$
\mathfrak{g}=\mathfrak{h} \oplus\left(\bigoplus_{\alpha \in R} \mathfrak{g}_{\alpha}\right)
$$

where $R$ is the root system associated to $\mathfrak{h}$. Since $\mathfrak{g}$ is simple, each $\mathfrak{g}_{\alpha}$ is one dimensional. Choose and fix a generic "oriented" hyperplane in $\mathfrak{h}^{*}$, and denote by $R_{+}$the set of positive roots relative to this hyperplane. For any $\alpha \in R_{+}$, let $e_{\alpha} \in \mathfrak{g}_{\alpha}$ and $f_{\alpha} \in \mathfrak{g}_{-\alpha}$ be nonzero vectors. Set $h_{\alpha}:=\left[e_{\alpha}, f_{\alpha}\right]$. Then $\mathfrak{s l}_{\alpha}=$ span $\left\{e_{\alpha}, h_{\alpha}, f_{\alpha}\right\}$ is a subalgebra of $\mathfrak{g}$ which is isomorphic to $\mathfrak{s l}(2, \mathbb{C})$, and $e_{\alpha}, h_{\alpha}$ and $f_{\alpha}$, can be rescaled so as to satisfy the canonical commutation relations. We denote by $E_{\alpha}, H_{\alpha}$ and $F_{\alpha}$ the vector fields $\vec{e}_{\alpha}, \vec{h}_{\alpha}$ and $\vec{f}_{\alpha}$ on End $\mathfrak{g}$ defined above.

Without loss of generality, we may assume that $x_{1}$ and $x_{2}$ are any of $e_{\alpha}, h_{\alpha}, f_{\alpha}$ where $\alpha \in R_{+}$. Moreover, because of the symmetry between the $e_{\alpha} \mathrm{s}$ and $f_{\alpha} \mathrm{s}$, it suffices to prove only that

$\left(h_{\alpha} \otimes \xi\right) E_{\beta}, \quad\left(h_{\alpha} \otimes \xi\right) H_{\beta}, \quad\left(f_{\alpha} \otimes \xi\right) E_{\beta}, \quad\left(e_{\alpha} \otimes \xi\right) H_{\beta}, \quad$ and $\quad\left(e_{\alpha} \otimes \xi\right) E_{\beta}$

are generated by overshears. In what follows, $n$ will be used to denote some integer which may vary from case to case. Moreover, we constantly use Proposition 6.2 without reference.

1: $\left(h_{\alpha} \otimes \xi\right) H_{\beta}$ is a shear and $\left(h_{\alpha} \otimes \xi\right) E_{\beta}$ an overshear.

Proof.

$$
\begin{aligned}
H_{\beta}\left(h_{\alpha} \otimes \xi\right) & =\left[h_{\beta}, h_{\alpha}\right] \otimes \xi=0 . \\
E_{\beta}^{2}\left(h_{\alpha} \otimes \xi\right) & =E_{\beta}\left(\left[e_{\beta}, h_{\alpha}\right] \otimes \xi\right) \\
& =E_{\beta}\left(n e_{\beta} \otimes \xi\right) \\
& =n\left[e_{\beta}, e_{\beta}\right] \otimes \xi=0
\end{aligned}
$$

2: $\left(f_{\alpha} \otimes \xi\right) E_{\beta}=\frac{1}{2}\left(\left[F_{\alpha},\left(h_{\alpha} \otimes \xi\right) E_{\beta}\right]+n\left(h_{\alpha} \otimes \xi\right) H_{\beta}\right)$, so in view of Case $1,\left(f_{\alpha} \otimes \xi\right) E_{\beta}$ is generated by overshears.

3: $\left(e_{\alpha} \otimes \xi\right) H_{\beta}=-\frac{1}{2}\left(\left[E_{\alpha},\left(h_{\alpha} \otimes \xi\right) H_{\beta}\right]+n\left(h_{\alpha} \otimes \xi\right) E_{\beta}\right)$, so in view of Case $1,\left(e_{\alpha} \otimes \xi\right) H_{\beta}$ is generated by overshears.

4: $\left(e_{\alpha} \otimes \xi\right) E_{\beta}=-\frac{1}{2}\left(\left[E_{\beta},\left(e_{\alpha} \otimes \xi\right) H_{\beta}\right]+c\left(e_{\alpha+\beta} \otimes \xi\right) H_{\beta}\right)$ for some constant $c$, so in view of Cases 1 and $3,\left(e_{\alpha} \otimes \xi\right) H_{\beta}$ is generated by overshears.

This completes the proof. 


\section{The general case}

In this section we will prove our main result.

Theorem 7.1. Every complex semisimple Lie group has the density property.

Most of the ingredients of the proof are the same as those used in Theorem 6.3 and the theorems of Sect. 4; if $G$ is a complex semisimple Lie group, we find a faithful representation $V$ of $G$ which embeds $G$ as a closed subgroup of $S L(V)$, and use the "right invariant scheme" introduced several times above. The key point of the proof, already used implicitly in the proof of Theorem 6, can then be roughly sketched as follows: if you are in the situation of Theorem 5.1 and have an $\mathfrak{s l}(2, \mathbb{C})$-algebra generated by weight vectors having weights between -2 and +2 which are either killed by or permuted by the $\mathfrak{s l}(2, \mathbb{C})$-action, then the vector field algebra associated to this $\mathfrak{s l}(2, \mathbb{C})$-algebra is generated by overshears; the even weight vectors are taken care of by Theorem 3.5, and one needs only to prove that if $x$ has weight \pm 1 , then $x \otimes E, x \otimes H$ and $x \otimes F$ are completely generated. The latter is done exactly as in the proof of Theorem 6.3. Thus the only thing left to do is to show that $G$ has a representation whose weights facilitate this technique. We now make this point precise, and produce the representation of $G$ alluded to.

Let $G$ be a semisimple Lie group, and let $V$ be a representation of $G$. Then $G$ is a closed subgroup of $S L(V)$. The representation $V$ induces a representation on $E n d V \cong V \otimes V^{*}$ given by

$$
g(v \otimes \xi)=(g v) \otimes \xi
$$

Analytically, $G$ is properly holomorphically embedded in $V \otimes V^{*}$, and hence (the restrictions to $G$ of) the algebra $\mathbb{C}\left[V \otimes V^{*}\right]$ of polynomials on $V \otimes V^{*}$ is dense in $\mathcal{O}(G)$. We identify $\mathbb{C}\left[V \otimes V^{*}\right]$ with $S\left(V \otimes V^{*}\right)$, the symmetric algebra generated by $V \otimes V^{*}$, (this amounts to identifying EndV with its dual) and extend the action of $G$ to $S\left(V \otimes V^{*}\right)$ naturally:

$$
g p(v \otimes \xi):=p(g v \otimes \xi) .
$$

As above, this induces (via differentiation, say) a representation of the Lie algebra $\mathfrak{g}$ of $G$ on End $V$ which is tangent to $G$, acts on $v \otimes \xi$ by $X(v \otimes \xi)=(X v) \otimes \xi$, and extends to $S\left(V \otimes V^{*}\right)$ by the Leibniz rule. Finally it is worth noting that $\mathfrak{g}$ is in fact realized as the Lie algebra of right invariant vector fields on $G$, naturally extended to $V \otimes V^{*}$.

Our goal now is to find a representation $V$ of $G$ so that the above realization of $G$ as a submanifold of $V \otimes V^{*}$ satisfies the criterion formulated at the beginning of Sect. 5 . We shall now define a sufficiently broad class of such representations. Recall that $\mathfrak{g}$, being semisimple, has the property that it is the linear span of its $\mathfrak{s l}(2, \mathbb{C})$-subalgebras. Let us fix a Cartan subalgebra $\mathfrak{h}$ of $\mathfrak{g}$ and let $R$ be the corresponding root system in $\mathfrak{h}$. 
Definition 7.2. We say that a representation $V$ of $G$ is bounded by 2 if it is generated by weight vectors $v_{1}, \ldots, v_{N}$ with the following property: if for each $\alpha \in R$ we restrict the representation to the subalgebra $\mathfrak{s l}_{\alpha}:=$ $\mathfrak{g}_{\alpha} \oplus \mathfrak{h} \oplus \mathfrak{g}_{-\alpha}$ of $\mathfrak{g}$, then all the weights appearing in the representation $V$ of $\mathfrak{s l}_{\alpha}$ lie in $\{0, \pm 1, \pm 2\}$.

Proposition 7.3. Let $G$ be a semisimple Lie group admitting a faithful representation $V$ which is bounded by 2 . Then $G$ has the density property.

Proof. By Theorem 5.1 it suffices (using the construction above) to show that for each $X \in \mathfrak{g}$ (thought of as a vector field on $G$ ) and $v \otimes \xi \in V \otimes V^{*}$, $(v \otimes \xi) \cdot X$ is completely generated. We may assume that $v$ is a weight vector, and that $X$ is a weight vector relative to some Cartan subalgebra $\mathfrak{h}$ of $\mathfrak{g}$. Since in this case $X$ lies in an $\mathfrak{s l}(2, \mathbb{C})$-subalgebra $\operatorname{span}\{E, H, F\}$ of $\mathfrak{g}$, we may further assume that $X=E$ or $H$. Moreover, by Theorem 3.5, we may assume that $v$ has weight \pm 1 with respect to $H$.

$X=E$ : Then $X^{2} v$ has weight $\geq 3$, and hence must vanish. Thus $X^{2} v \otimes \xi=0$, so $(v \otimes \xi) \cdot X$ is an overshear.

$X=H$ : Then $(v \otimes \xi) \cdot H=[(v \otimes \xi) \cdot E, F]+(F v \otimes \xi) \cdot E$. Now, either $F v$ has weight -1 or it vanishes. Either way, using the previous case, $(v \otimes \xi) \cdot H$ is generated by overshears.

This completes the proof.

In view of Proposition 7.3, Theorem 7.1 follows immediately from the following result.

Theorem 7.4. Every complex semisimple Lie group admits a faithful representation which is bounded by 2 .

For the reader who would like to recall the main ideas of the representation theory of semisimple Lie algebras, or who is unfamiliar with the notation used here, we recommend chapter 14 of $[\mathrm{FH}]$. For a well organized collection of the data used below, we recommend [B], Planches I-IX.

Again, we fix a complex semisimple Lie group $G$ with Lie algebra $\mathfrak{g}$, and choose a Cartan subalgebra $\mathfrak{h}$ of $\mathfrak{g}$. Let $R$ denote the corresponding root system, and denote by $\Lambda_{R}$ (resp. $\Lambda_{W}$ ) the root lattice (resp. weight lattice) in $\mathfrak{h}^{*}$. We now construct a special set of dominant weights $L=$ $\left\{\lambda_{1}, \ldots, \lambda_{r}\right\} \subset \Lambda_{W}$ which surjects onto $\Lambda_{W} / \Lambda_{R}$.

Let $S=\left\{\alpha_{1}, \ldots, \alpha_{n}\right\}$ be a base, or set of simple roots, of $R$, and let $W=\left\{\omega_{1}, \ldots, \omega_{n}\right\}$ be the corresponding set of fundamental weights, i.e., those $\omega_{j} \in \Lambda_{W}$ for which $\omega_{j}\left(H_{\alpha_{k}}\right)=\delta_{j k}$. (Recall that $H_{\alpha}$ is defined by the two conditions (i) $H_{\alpha} \in\left[\mathfrak{g}_{\alpha}, \mathfrak{g}_{-\alpha}\right]$ and (ii) $\alpha\left(H_{\alpha}\right)=2$ ).) 
For the simple Lie algebras, we define $L$ as follows:

- $R$ is of type $A_{l}: L=W \cup\left\{\omega_{1}+\omega_{l}\right\}$,

- $R$ is of type $B_{l}: L=\left\{\omega_{1}, \omega_{2}\right\}$,

- $R$ is of type $C_{l}: L=\left\{\omega_{1}, \omega_{2}\right\}$

- $R$ is of type $D_{l}: L=\left\{\omega_{1}, \omega_{2}, \omega_{l-1}, \omega_{l}\right\}$ if $l>3,\left\{\omega_{1}, \omega_{2}, \omega_{3}, \omega_{2}+\omega_{3}\right\}$ if $l=3$,

- $R$ is of type $E_{6}: L=\left\{\omega_{1}, \omega_{2}, \omega_{3}\right\}$,

- $R$ is of type $E_{7}: L=\left\{\omega_{1}, \omega_{2}\right\}$,

- $R$ is of type $E_{8} F_{4}$ or $G_{2}: L=\left\{\omega_{1}\right\}$.

If $\mathfrak{g}$ is not simple, we can write it as a sum of simple ideals $\mathfrak{g}=\mathfrak{g}_{1} \oplus \ldots \oplus \mathfrak{g}_{s}$ The Cartan can then be written as a sum of Cartans of the simple ideals, $\mathfrak{h}=\mathfrak{h}_{1} \oplus \ldots \oplus \mathfrak{h}_{s}$, and the corresponding root systems have an analogous decomposition $R=R_{1} \oplus \ldots \oplus R_{s}$. The base $S$ then has a decomposition $S=S_{1} \cup \ldots \cup S_{S}$ with $S_{i}$ a base of $R_{i}$, and the set of fundamental weights decomposes as $W=W_{1} \cup \ldots \cup W_{s}$. Of course if $i \neq j$, then for $\alpha \in S_{i}$ and $\omega \in W_{j}, \omega\left(H_{\alpha}\right)=0$. Our choice of $L$ is made as follows: To each simple $\mathfrak{g}_{i}$ there corresponds an $L_{i}$ according to the above list. We take the "sum" of the $L_{i}$ :

$$
L=\left\{\lambda^{(1)}+\ldots+\lambda^{(s)} ; \lambda^{(i)} \in L_{i}\right\} \subset L_{1} \oplus \ldots \oplus L_{s} .
$$

The following fact then holds: to each $\lambda \in L$ there corresponds a representation of $\mathfrak{g}$ which is bounded by 2 . Of course, it suffices to check this fact for the roots systems of simple Lie algebras, i.e., to compute the integers $\lambda^{(i)}\left(H_{\alpha}\right), \alpha \in R_{i}$ and to show that these numbers always lie between +2 and -2 . The latter can be verified directly using Planches I-IX of [B].

Proof of Theorem 7.4. Consider the representation which is the direct sum of all the representations of $G$ arising from the (dominant) weights in $L \cap \Lambda_{G}$. Although not irreducible, this representation is faithful, and by its very construction is bounded by 2 .

Remark. The choices of the $L_{i}$ above are in no way unique; there are many other ways to choose them which will still produce faithful representations that are bounded by 2 .

\section{References}

[A] Andersén, E., Volume preserving automorphisms of $\mathbb{C}^{n}$. Complex Variables 14, 223-235 (1990)

[AL] Andersén, E., Lempert, L., On the group of holomorphic automorphisms of $\mathbb{C}^{n}$. Invent. Math. 110, 371-388 (1992)

[B] Bourbaki, N., Éléments de mathématique. (French) [Elements of mathematics] Groupes et algébres de Lie. Chapitres 4, 5 et 6. [Lie groups and Lie algebras. Chapters 4, 5 and 6] Masson, Paris, 1981. 290 pp.

[F] Forstnerič, F., Holomorphic Automorphisms of $\mathbb{C}^{n}$ : A Survey. (Proceedings 'Complex Analysis and Geometry', Ed. V. Ancona, E. Ballico, A. Silva; pp. 173-200) Lect. Notes Pure Appl. Math. 173, New York: Marcel Dekker (1996) 
[FR] Forstnerič, F., Rosay, J.P. Approximation of biholomorphic mappings by automorphisms of $\mathbb{C}^{n}$. Invent. Math. 112, 323-349 (1993)

$[\mathrm{FH}]$ Fulton, W., Harris, J., Representation Theory, A First Course, Graduate Texts in Mathematics, 129. Readings in Mathematics. Springer-Verlag, New York, 1991. $\mathrm{xvi}+551 \mathrm{pp}$.

[R] Rosay, J.-P., Automorphisms of $C^{n}$, a survey of Andersén-Lempert theory and applications. Complex geometric analysis in Pohang (1997), 131-145, Contemp. Math. 222, Amer. Math. Soc., Providence, RI (1999)

[RR] Rosay, J.P., Rudin, W., Holomorphic maps from $\mathbb{C}^{n}$ to $\mathbb{C}^{n}$. Trans. AMS 310, 47-86 (1988)

[V1] Varolin, D., Complex manifolds and geometric structures with the density property, to appear in J. Geom. Anal.

[V2] Varolin, D., Complex manifolds and geometric structures with the density property II, preprint 1997

[V3] Varolin, D., A general notion of shears, and applications, to appear in Mich. Math. J.

[W] Winkelmann, J., Large discrete sets in Stein manifolds, preprint 1998 